\title{
Baroreflex sensitivity is depressed in microalbuminuric Type I diabetic patients at rest and during sympathetic manoeuvres
}

\author{
J.D. Lefrandt ${ }^{1}$, K. Hoogenberg ${ }^{2}$, A.M. van Roon ${ }^{1}$, R.P. F. Dullaart ${ }^{2}$, R. O. B. Gans ${ }^{1}$, A. J. Smit ${ }^{1}$ \\ ${ }^{1}$ Department of Internal Medicine, Division of Angiology and General Medicine, State University Hospital, Groningen, \\ The Netherlands \\ ${ }^{2}$ Department of Internal Medicine, Division of Endocrinology, State University Hospital, Groningen, The Netherlands
}

\section{Abstract}

Aims/hypothesis. To evaluate baroreflex sensitivity (BRS) in microalbuminuric and normoalbuminuric Type I (insulin-dependent) diabetic patients without autonomic neuropathy and in healthy control subjects.

Methods. Microalbuminuric Type I diabetic patients $(n=15)$ were matched for age, sex, body mass index (BMI) and smoking habits with 15 normoalbuminuric patients and with 15 healthy control subjects. All subjects had a blood pressure less than $160 / 95 \mathrm{mmHg}$, a BMI less than $30 \mathrm{~kg} / \mathrm{m}^{2}$ and normal autonomic function on standard tests. Blood pressure and heart rate were measured non-invasively (Finapres) at rest and during sympathetic activation (handgrip, mental stress, standing). The baroreflex sensitivity was defined as the mean gain between blood pressure variability and heart rate variability in the $0.07-0.15 \mathrm{~Hz}$ frequency band.
Results. Resting baroreflex sensitivity was decreased in the microalbuminuric patients $(3.5 \pm 0.4 \mathrm{~ms} /$ $\mathrm{mmHg}$ ) compared with the normoalbuminuric patients and the healthy subjects $(7.6 \pm 1.6$ and $9.5 \pm 1.1 \mathrm{~ms} / \mathrm{mmHg}$, respectively, $p<0.001)$. The sympathetic tests reduced baroreflex sensitivity similarly in the groups without changing the between group differences.

Conclusion/interpretation. Baroreflex sensitivity is reduced in Type I diabetic patients with microalbuminuria but without autonomic neuropathy. A prospective study should indicate whether this early abnormality in cardiovascular reflex function is a risk factor of cardiovascular mortality in these patients. [Diabetologia (1999) 42: 1345-1349]

Keywords Cardiovascular autonomic function, diabetes mellitus, microalbuminuria, heart rate variability, blood pressure variability, baroreflex sensitivity.
The presence of microalbuminuria indicates an increased risk of cardiovascular disease in patients with Type I (insulin-dependent) diabetes mellitus [1]. Clinical autonomic neuropathy adds to a particular poor prognosis in these patients [2-5]. Because autonomic neuropathy is highly prevalent among pro-

Received: 20 May 1999 and in revised form: 8 July 1999

Corresponding author: K. Hoogenberg, MD, PhD, Department of Internal Medicine, Division of Endocrinology, University Hospital Groningen, P.O. Box 30.001, 9700 RB Groningen, The Netherlands.

Abbreviations: BRS, Baroreflex sensitivity; ACE, angiotensin converting enzyme. teinuric patients, it could in part account for the increased mortality rate associated with diabetic nephropathy [6, 7]. In addition, autonomic neuropathy could play a part in the development and the progression of proteinuria [8]. Abnormal cardiovascular reflexes predict a lower than expected glomerular filtration rate $[9,10]$ and the presence of autonomic neuropathy blunts the nocturnal fall in blood pressure and albumin excretion rate [11].

Baroreflex sensitivity (BRS) measurements are highly sensitive for detecting early abnormalities in cardiovascular autonomic function $[12,13]$. Baroreflex sensitivity has been reported to be impaired in uncomplicated Type I diabetic patients [13-15] but the clinical relevance of a decline in BRS in these pa- 
Table 1. Clinical characteristics of the study groups

\begin{tabular}{|c|c|c|c|}
\hline & $\begin{array}{l}\text { Microalbuminuric } \\
\text { diabetic patients }\end{array}$ & $\begin{array}{l}\text { Normoalbuminuric } \\
\text { diabetic patients }\end{array}$ & Control subjects \\
\hline Number & 15 & 15 & 15 \\
\hline Age (years) & $50 \pm 3$ & $49 \pm 3$ & $47 \pm 3$ \\
\hline Sex (men/women) & $11 / 4$ & $11 / 4$ & $11 / 4$ \\
\hline Cigarette smokers $(n)$ & 4 & 4 & 4 \\
\hline Body mass index $\left(\mathrm{kg} / \mathrm{m}^{2}\right)$ & $26.3 \pm 0.8$ & $24.9 \pm 0.6$ & $24.2 \pm 0.8$ \\
\hline Diastolic blood pressure (mm Hg) & $75 \pm 2$ & $73 \pm 3$ & $79 \pm 2$ \\
\hline Pulse rate (beats/min) & $78 \pm 3^{b}$ & $67 \pm 3$ & $67 \pm 3$ \\
\hline Serum creatinine $(\mu \mathrm{mol} / \mathrm{l})$ & $88 \pm 6$ & $84 \pm 3$ & $85 \pm 3$ \\
\hline Urinary albumin excretion (ug/min) & $54.9 \pm 9.6^{c}$ & $4.3 \pm 0.6$ & $3.9 \pm 0.6$ \\
\hline Glycated haemoglobin (\%) & $8.2 \pm 0.2$ & $7.9 \pm 0.2$ & $5.4 \pm 0.1^{\mathrm{d}}$ \\
\hline Insulin dose $\left(\mathrm{U} \cdot \mathrm{kg}^{-1} \cdot \mathrm{day}^{-1}\right)$ & $0.8 \pm 0.1$ & $0.7 \pm 0.1$ & - \\
\hline
\end{tabular}

Values are given in means \pm SEM, except urinary albumin excretion rate which is given in geometric means \pm antilog SEM (not normally distributed). Retinopathy A: absent, B: background, P: proliferative; ${ }^{a} p<0.05$ vs normoalbuminuric dia-

tients is not yet clear. It could be a risk indicator of cardiovascular mortality, in analogy to observations in post-myocardial patients $[16,17]$.

An increase in the carotic wall thickness [18] and a reduced distensibility [19] have both been reported in microalbuminuric Type I diabetic patients and could affect the function of baroreceptors in the carotic wall. An impaired cardiac vagal function [6] and a diminished endothelial function [20] are other abnormalities reported in microalbuminuric Type I diabetic patients that could also contribute to an abnormal baroreflex function in these patients.

In our study, we evaluated the possibility of an abnormal baroreflex function in microalbuminuric Type I diabetic patients. Therefore, we assessed BRS in matched microalbuminuric and normoalbuminuric patients without autonomic neuropathy and healthy subjects at rest and during sympathetic manoeuvres.

\section{Subjects and methods}

Subjects. All subjects consented to participate in the study, which was approved by the local medical ethics committee. Eligible subjects were aged between 30 and 65 years, had a serum creatinine less than $120 \mu \mathrm{mol} / \mathrm{l}$, a body mass index less than 30 $\mathrm{kg} / \mathrm{m}^{2}$, a systolic blood pressure less than $160 \mathrm{mmHg}$ and a diastolic blood pressure less than $95 \mathrm{mmHg}$ and no signs of autonomic neuropathy as assessed by standard tests [21]. Autonomic function was evaluated by the Valsalva manoeuvre (15 s breathing with $40 \mathrm{mmHg}$ counter-pressure), beat-to-beat variation during six cycles of maximum inspiration and expiration, the drop in systolic blood pressure and the 30:15 ratio of the change in heart rate after standing [21]. Results were com- betic patients; ${ }^{\mathrm{b}} p<0.02$ and ${ }^{\mathrm{c}} p<0.001$ vs normoalbuminuric diabetic patients and control subjects; ${ }^{\mathrm{d}} p<0.001$ vs normoalbuminuric and microalbuminuric diabetic patients pared with age-adjusted normalised values [22] and autonomic neuropathy was considered to be present when two or more tests were abnormal [21]. The diabetic patients were considered insulin-dependent because of ketosis prone diabetes and a disease onset before 35 years of age. In case of doubt, insulin deficiency was confirmed by a glucagon-stimulated plasma $\mathrm{C}$ concentration peptide less than $0.2 \mathrm{nmol} / \mathrm{l}$. They had a duration of disease of at least 10 years and were categorised for the presence of microalbuminuria. Microalbuminuria was defined as an urinary albumin excretion rate between 20 and $200 \mu \mathrm{g} /$ min in at least two of the three overnight urine collections in the preceeding year. Patients with a history of coronary heart disease, peripheral vascular disease and those who used betablockers or calcium channel-blockers were excluded.

We studied 15 microalbuminuric, 15 normoalbuminuric Type I diabetic patients and 15 healthy control subjects. They were matched for sex, age (within 5 years), body mass index (within $2.5 \mathrm{~kg} / \mathrm{m}^{2}$ ) and smoking habits (Table 1). Metabolic control and daily insulin requirement was similar in the normoalbuminuric and microalbuminuric diabetic groups. Systolic and diastolic blood pressure were not essentially different between the groups although pulse rate was higher in the microalbuminuric than in normoalbuminuric patients and healthy control subjects $(p<0.02$, Table 1$)$. We studied five microalbuminuric and two normoalbuminuric patients while using an angiotensin converting enzyme (ACE) inhibitor. Of these, three microalbuminuric and one normoalbuminuric patients were additionally treated with diuretics. Because of their participation in a study requiring discontinuation of medication ten microalbuminuric patients stopped their ACE-inhibition or diuretic mediations or both 4 to 6 weeks before the measurements.

Baroreflex sensitivity measurements during rest and sympathetic activation. The studies were done $2 \mathrm{~h}$ after breakfast at which the patients had taken their usual morning insulin dose. The measurements were carried out in a quiet temperaturecontrolled $\left(22^{\circ} \mathrm{C}\right)$ room with the participants in the supine position. Smoking and caffeine bearing drinks were not allowed 
during the study day. After a 30-min rest, a Finapres cuff (Finapres, Ohmeda 2300, Englewood, Colo., USA) was applied to the midphalanx of the third finger for continuous blood pressure and heart rate monitoring. After 20 min of baseline measurements, the subjects underwent a further battery of autonomic function tests. These included isometric handgrip ( 3 min isometric contraction at $30 \%$ of maximum strength), mental stress (3 min of arithmetic calculations) and standing (5 min standing in upright position).

Baroreflex sensitivity was determined by the transfer function technique using the CARSPAN program, as described $[23,24]$. This program allows discrete Fourier transformation of non-equidistant samples of blood pressure and RR interval series. The analysed time-series are corrected for artefacts and checked for stationarity. Baroreflex sensitivity is defined as the mean modulus between spectral values of systolic blood pressure variability and heart rate variability in the $0.07-0.15$ $\mathrm{Hz}$ frequency band with a coherence of more than 0.3 . Baroreflex sensitivity is expressed in $\mathrm{ms} / \mathrm{mmHg}$. A BRS of $10 \mathrm{~ms} /$ $\mathrm{mmHg}$ indicates that a rise of $1 \mathrm{mmHg}$ in systolic blood pressure will induce $10 \mathrm{~ms}$ of RR interval lengthening. The coefficient of variation of this method is $13 \%$. In this study, resting BRS was determined from three periods of 100 to $300 \mathrm{~s}$. Modulation of BRS in response to the sympathetic tests was assessed from a stationary segment of at least $100 \mathrm{~s}$.

Statistical analysis. Power spectral values have a skewed distribution that is normalised after logarithmic transformation. Therefore, the natural logarithm of BRS is used in the analyses. Within and between group differences were analysed with ANOVA and post-hoc analysis with Bonferroni adjustment for multiple comparisons. Correlation were sought with Pearson's tests, the independent contribution of variables was evaluated with multiple regression analysis. Data are given as means \pm SEM, unless stated otherwise. The findings of Valsalva manoeuvre, beat-to-beat variation on deep breathing, 30:15 ratio and drop in systolic blood pressure after standing were ranked separately and the average rank was used as an arbitrary measure of overall autonomic function. A $p$ value of less than 0.05 was considered significant.

\section{Results}

Resting supine BRS was reduced in microalbuminuric patients compared with normoalbuminuric patients and healthy control subjects ( $p<0.001$, Fig. 1$)$. There was no numerical difference in BRS between diabetic patients studied with and without ACE-inhibition therapy (Fig.1). Isometric handgrip, mental stress and standing induced similar changes in systolic pressure in the three groups (change in systolic blood pressure during handgrip: $35.4 \pm 5.7,31.6 \pm 4.1$, $28.8 \pm 3.4 \mathrm{mmHg}$; during mental stress: $28.5 \pm 3.3$, $25.8 \pm 2.6,25.3 \pm 3.2 \mathrm{mmHg}$ in the microalbuminuric, and normoalbuminuric diabetic patients and healthy control subjects, respectively, $p<0.001$ for all; during standing: Table 1). Baroreflex sensitivity decreased during isometric handgrip and to standing, increased post-standing ( $p<0.01$ in all groups, Fig. 2$)$ but was not significantly altered by mental stress. The changes in BRS during handgrip, standing and post-standing did not differ between the groups (Fig. 2).

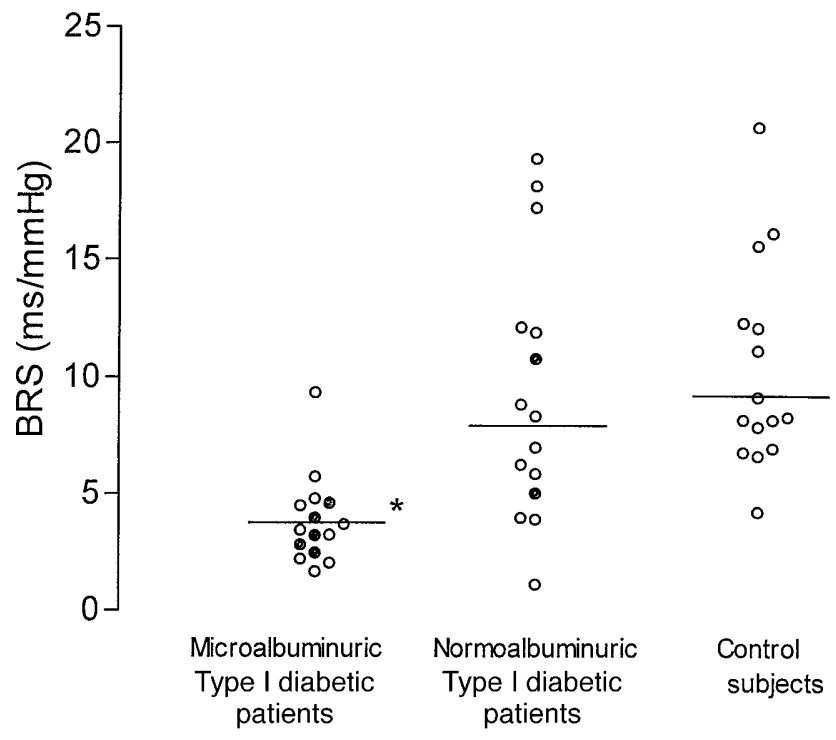

Fig. 1. Individual resting supine BRS measurements in microalbuminuric, normoalbuminuric Type I diabetic patients and healthy control subjects. • measurements of patients using an angiotensin converting enzyme-inhibitor. Horizontal lines indicate geometric means. $* p<0.001$ vs normoalbuminuric patients and healthy control subjects

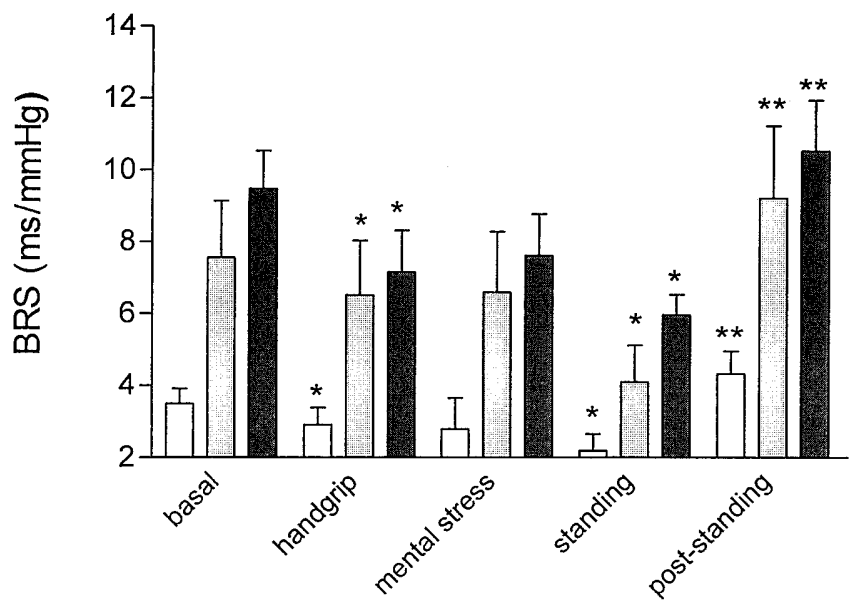

Fig. 2. Baroreflex sensitivity measurements at rest, isometric handgrip, mental stress, standing and after standing in the micoalbuminuric $(\square)$, the normoalbuminuric $(\square)$ Type I diabetic patients and the healthy control subjects ( Data are represented as geometric means \pm antilog SEM. $* p<0.01$ from resting supine BRS and $* * p<0.01$ from standing BRS

Baseline BRS correlated significantly with age $(r=-0.46)$, diabetes duration $(r=-0.54), \quad \mathrm{HbA}_{1 \mathrm{c}}$ $(r=-0.42)$, BMI $(r=-0.40)$, overall autonomic function test score $(r=-0.52)$, grade of retinopathy (defined as absent, background or proliferative) $(r=0.57)$, heart rate $(r=-0.58)$, systolic blood pressure $(r=-0.48)$ and urinary albumin excretion rate $(r=0.57, p<0.01$ for all). Multiple regression analysis showed that resting heart rate contributed to 
$15 \%(p<0.001)$, urinary albumin excretion rate to $14 \%(p<0.001)$, age to $13 \%(p<0.001)$ and overall autonomic function to $7 \%(p<0.05)$ to the variance in BRS (multiple $r=0.70, p<0.001$ ), without significant contributions of diabetes duration, $\mathrm{HbA}_{1 \mathrm{c}}$, grade of retinopathy and systolic blood pressure (all $p>0.20)$.

Furthermore, multiple regression analysis showed that the Valsalva ratio $(r=0.45, p<0.001)$, beat-tobeat variation during deep breathing $(r=0.46$, $p<0.001)$ and $30: 15$ ratio after standing $(r=0.55$, $p<0.001)$ were explained only by the baseline BRS measurements without statistically significant contributions of other clinical variables. Baseline BRS was not related to the blood pressure responses at handgrip, mental stress and standing testing.

\section{Discussion}

In our study, baroreflex control of blood pressure was reduced in Type I diabetes mellitus complicated by microalbuminuria but still showed the ability to modulate in response to sympathetic stress tests. The impaired BRS indicates that autonomic dysfunction is present at an early stage of diabetic renal involvement even when there is no clinical autonomic neuropathy as defined by accepted criteria [21].

In a multiple regression analysis, we found that microalbuminuria independently from well-known other factors that influence BRS, like age and autonomic function [13-15], contributed to the reduction in BRS. Thus, the presence of microalbuminuria seems to be an important factor to take into consideration in BRS abnormalities of Type I diabetic patients. We did not find a numerical difference in baseline BRS between diabetic patients with and without ACE-inhibition therapy.

We can only speculate on the clinical relevance of the association between microalbuminuria and reduced BRS in the Type I diabetic patients. The aim of the baroreflex is short-term blood pressure control by modulation of vagal and sympathetic influence on heart rate, cardiac contractility and peripheral vascular resistance. A sensitive system effectively responds to small rises in blood pressure with an increase in vagal activity and a decrease in sympathetic tone, resulting in a deceleration of heart rate, a diminished contractility and a reduction of peripheral resistance. A drop in blood pressure causes opposite responses. Baroreflex sensitivity is a marker of the capability of the system to buffer blood pressure variations and a reduction in BRS has been found to be highly predictive of mortality in post-myocardial infarction patients $[16,17]$. The increased mortality rate of patients with a low BRS is attributed to an increased vulnerability to develop lethal arrhythmias $[16,17]$. Based on the observations in post-myocardial infarc- tion patients, we suggest our finding of a reduced BRS in conjunction with the presence of microalbuminuria in Type I diabetic patients could similarly indicate an increased cardiovascular risk. Indeed, the occurrence of microalbuminuria is associated with an increased risk of cardiovascular disease [1]. Moreover, a higher incidence of sudden cardiac deaths has been reported in diabetic patients [25]. A diminished BRS indicates an impaired responsiveness to blood pressure variations of the baroreflex system. Therefore, another consequence of the diminished BRS of the microalbuminuric Type I diabetic patients could be an impairment of short-term blood pressure control. We were, however, unable to show a changed blood pressure responsiveness or variability despite the use of sympathetic tests. This does not exclude the possibility that microalbuminuric Type I diabetic patients are prone to larger blood pressure excursions during daily life activities when BRS is impaired. The suggestion warrants further investigation as a decreased systemic blood pressure buffering capacity could be of importance in patients with impaired renal haemodynamics [26].

Baroreflex sensitivity was not essentially lower in the normoalbuminuric Type I diabetic patients compared with the healthy control subjects, despite some of these patients having distinct abnormal values. This finding is at variance with recent observations that showed a decreased BRS in uncomplicated Type I diabetic patients [13-15]. The methods to calculate BRS differed, however, among these studies, as the $\alpha$-method [13], the sequential technique [13-15] and the transfer function method [present study] were used. Despite these techniques having all been validated against classical pharmacological BRS assessments [24, 27], the impact of the differences in BRS outcome in diabetic patients awaits further evaluation. The conclusion that BRS measurements unequivocally detect early abnormalities in cardiovascular autonomic function [12-15] is illustrated by the findings of multiple regression analysis in which only the BRS, independent from other factors, contributed to the variance of the standard autonomic function tests. It is generally thought that standard autonomic function tests are hampered by a low discriminative power to detect abnormalities in cardiovascular autonomic function $[6,8,16]$. Despite the accuracy in measuring early abnormalities, modern analyses of cardiovascular rhythms and reflexes, like BRS assessments, have not yet been documented to be a risk factor of mortality in diabetic patients. This contrasts with the well-documented power of autonomic function tests to predict mortality in diabetic patients [2-5].

In conclusion, BRS is reduced in Type I diabetic patients complicated by microalbuminuria. Further investigations are warranted to assess the possible predictive power of a reduced BRS on mortality in these patients. 
Acknowledgements. We are indebted to M. Bruin, B. Buist, A. van Gessel, W. Kuipers and M. Teune for their skilful laboratory assistance.

\section{References}

1. Messent JW, Elliott TG, Hill RD, Jarrett RJ, Keen H, Viberti GC (1992) Prognostic significance of microalbuminuria in insulin-dependent diabetes mellitus: a twenty-three year follow-up study. Kidney Int 41: 836-839

2. Ewing DJ, Campbell IW, Clarke BF (1976) Mortality in diabetic autonomic neuropathy. Lancet i:601-603

3. O'Brien IA, McFadden JP, Corrall RJM (1991) The influence of autonomic neuropathy on mortality in insulin-dependent diabetes. QJM 79: 495-502

4. Rathmann W, Ziegler D, Jahnke M, Haastert B, Gries FA (1993) Mortality in diabetic patients with cardiovascular autonomic neuropathy. Diabet Med 10: 820-824

5. Reichard P, Pihl M (1994) Mortality and treatment side-effects during long-term intensified conventional insulin treatment in the Stockholm Diabetes Intervention Study. Diabetes 43: 313-317

6. Mølgaard H, Christensen PD, Hermansen K, Serensen KE, Christensen KE, Mogensen CE (1994) Early recognition dysfunction in microalbuminuria: significance for cardiovascular mortality in diabetes mellitus? Diabetologia 37: 788-796

7. Sawicki PT, Dahne R, Bender R, Berger M (1996) Prolonged QT interval as a predictor of mortality in diabetic nephropathy. Diabetologia 39: 77-81

8. Spallone V, Menzinger G (1997) Diagnosis of cardiovascular autonomic neuropathy in diabetes. Diabetes 46:[Suppl 2] S67-S76

9. Sundkvist G, Lilja B (1993) Autonomic neuropathy predicts deterioration in glomerular filtration rate in patients with IDDM. Diabetes Care 16: 773-779

10. Weinrauch LA, Kennedy FP, Gleason RE, Keough J, D'Elia JA (1998) Relationship between autonomic function and progression of renal disease in diabetic proteinuria: clinical correlations and implications for blood pressure control. Am J Hypertens 11: 302-308

11. Spallone V, Gambardella S, Maiello MR, Barini A, Frontoni S, Menzinger G (1994) Relationship between autonomic neuropathy, 24-h blood pressure profile and nephropathy in normotensive IDDM patients. Diabetes Care 17: 578-584

12. De Ferrari GM, Landolina M, Mantica M, Manfredini R, Schwartz PJ, Lotto A (1995) Baroreflex sensitivity, but not heart rate variability, is reduced in patients with life-threatening ventricular arrhythmias long after myocardial infarction. Am Heart J 130: 473-480
13. Frattola A, Parati G, Gamba P, Paleari F, Mauri G, DiRienzo M, Castiglioni P, Mancia G (1997) Time and frequency domain estimates of spontaneous baroreflex sensitivity provide early detection of autonomic dysfunction in diabetes mellitus. Diabetologia 40: 1470-1475

14. Weston PJ, James MA, Panerai R et al. (1996) Abnormal baroreceptor-cardiac reflex sensitivity is not detected by conventional tests of autonomic function in patients with insulin-dependent diabetes mellitus. Clin Sci (Colch)91: 59-64

15. Weston PJ, Panerai RB, McCullough A et al. (1996) Assessment of baroreceptor-cardiac reflex sensitivity using time domain analysis in patients with IDDM and the relation to left ventricular mass index. Diabetologia 39: 1385-1391

16. Farrell TG, Odemuyiwa O, Bashir Yet al. (1992) Prognostic value of baroreflex sensitivity testing after acute myocardial infarction. Br Heart J 67: 129-137

17. LaRovere MT, Bigger JT, Marcus FI, Mortara A, Schwartz PJ (1998) Baroreflex sensitivity and heart-rate variability in prediction of total cardiac mortality after myocardial infarction. Lancet 351: 478-484

18. Frost D, Beischer W (1998) Determinants of carotic artery wall thickening in young patients with Type I diabetes mellitus. Diabet Med 15: 851-857

19. Lambert J, Smulders RA, Aarsen RVT, Donker AJM, Stehouwer CDA (1998) Carotic artery stiffness is increased in microalbuminuric IDDM patients. Diabetes Care 21: 99-103

20. Jensen T, Bjerre-Knudsen J, Feldt-Rasmussen B, Deckert $\mathrm{T}$ (1989) Features of endothelial dysfunction in early diabetic nephropathy. Lancet i: 461-463

21. Ewing DJ, Clarke BF (1982) Diagnosis and management of diabetic autonomic neuropathy. B MJ 285: 916-918

22. Piha SJ (1991) Cardiovascular autonomic reflex tests: normal responses and age related reference values. Clin Physiol 11: 277-290

23. Robbe HW, Mulder LJ, Ruddel H, Langewitz WA, Veldman JB, Mulder G (1997) Assessment of baroreceptor reflex sensitivity by means of spectral analysis. Hypertension. 10: $538-543$

24. Saul JP, Berger RD, Albrecht P, Stein SP, Chen MH, Cohen RJ (1991) Transfer function analysis of the circulation: unique insights into cardiovascular regulation. Am J Physiol 261: H1231-H1245

25. Tattersall RB, Gill GV (1991) Unexplained death of Type I diabetic patients. Diabet Med 8: 49-58

26. Mogensen CE (1995) Diabetic renal disease: The quest for normotension and beyond. Diabet Med 12: 756-769

27. Watkins LL, Grossman P, Sherwood A (1996) Noninvasive assessment of baroreflex control in borderline hypertension. Hypertension 28: 238-243 